\title{
Effects of Phloroglucinol on In Vitro Methanogenesis, Rumen Fermentation, and Microbial Population Density
}

\author{
K.A. Sarwono ${ }^{a}$, M. Kondo ${ }^{a}$, T. Ban-Tokuda ${ }^{a}$, A. Jayanegara ${ }^{b}$, \& H. Matsui ${ }^{a *}$ \\ ${ }^{a}$ Graduate School of Bioresources, Mie University, \\ 1577 Kurimamachiya-cho, Tsu, Mie 514-8507, Japan \\ ${ }^{\mathrm{b}}$ Department of Nutrition and Feed Technology, Faculty of Animal Science, \\ IPB University (Bogor Agricultural University), \\ Kampus IPB Darmaga, Bogor 16680, Indonesia \\ *Corresponding author: matsui@bio.mie-u.ac.jp \\ (Received 14-11-2018; Revised 13-02-2019; Accepted 15-02-2019)
}

\begin{abstract}
This study investigated the effect of phloroglucinol (1,3,5-trihydroxybenzene) supplementation alone on methane production, rumen fermentation profiles, and microbial population structure of mixed in vitro cultures. Treatments included a control group containing a substrate with no supplement, and substrates supplemented with $2,4,6,8$, or $10 \mathrm{mmol} / \mathrm{L}$ of phloroglucinol. The results revealed that phloroglucinol was able to decrease methane production in a dose-dependent manner. The highest decrease was observed with 8 and $10 \mathrm{mmol} / \mathrm{L}$ supplementations. The relative quantity of methanogen was not affected by phloroglucinol, whereas genus Coprococcus was increased with increasing concentrations of phloroglucinol $(\mathrm{p}<0.05)$. Total gas production, dry matter digestibility (DMD), and $\mathrm{NH}_{3}-\mathrm{N}$ were significantly lowered by phloroglucinol $(\mathrm{p}<0.001)$. Total short-chain fatty acid (SCFA) concentration was not affected by phloroglucinol. Acetate proportion increased with the addition of phloroglucinol at the expense of propionate $(p<0.001)$. This might indicate the redirection of $[\mathrm{H}]$ from methane to acetate, and might be related to methane inhibition. Our study concluded that supplementation of phloroglucinol alone could decrease methane production by inhibiting nutrient digestibility in the rumen and by possible redirection of rumen fermentation to acetate production. Genus Coprococcus could be an important actor for phloroglucinol metabolism in the rumen.
\end{abstract}

Keywords: methane, methanogen, phloroglucinol, rumen

\section{INTRODUCTION}

Ruminants are one of the greatest sources of methane, a major greenhouse gas that poses a serious environmental threat, with 28- to 34-fold more global warming potential (GWP) than $\mathrm{CO}_{2}$ (Myhre et al., 2013). Rumen fermentation in the livestock sector contributes $2.12 \mathrm{Gt} \mathrm{CO}$ eq/year of methane, which is equal to $6.3 \%$ of the total global anthropogenic emission of greenhouse gases (Smith et al., 2014). Incremental increases in the atmospheric methane concentration enhance the greenhouse effect by trapping heat energy received from the sun in the Earth's atmosphere, resulting in global warming and leading to climate change. Besides its threat to the environment, methane also represents a loss of dietary energy in ruminants. Depending on the type of feed, ruminants loose between 2 and $12 \%$ of the gross energy intake from feed as methane (Johnson \& Johnson, 1995). Therefore, it is important to decrease methane emission from ruminants for both environmental and economic benefits.

Methane is a natural by-product of anaerobic fermentation in the rumen. Methane in the rumen is produced mainly through the hydrogenotrophic pathway using $\mathrm{CO}_{2}$ as the main carbon source and $\mathrm{H}_{2}$ as the main electron donor. Methane production serves as an electron sink to help rumen maintain low partial pressure of $\mathrm{H}_{2}$. Partial pressure of $\mathrm{H}_{2}$ plays an important role in rumen fermentation. High partial $\mathrm{H}_{2}$ pressure in the rumen inhibits ruminal fermentation of carbohydrates, the rate of microbial growth, and the synthesis of microbial protein (Knapp et al., 2014). A strategy to mitigate methane production by supplementing an alternative $[\mathrm{H}]$ sink in an effort to redirect rumen fermentation away from methanogenesis has been proposed (Broucek, 2018, Lan \& Yang, 2019). The use of dicarboxylic acids, such as fumarate and malate to decrease methane production in the rumen has been evaluated (Martin et al., 2010, Song et al., 2011, Li et al., 2018). These compounds were found to decrease methane production but increase short-chain fatty acid (SCFA) production, which is the main energy source of ruminant. Other compounds also have been found to lower methane production by redirecting $[\mathrm{H}]$ utilization, such as nitrate and sulfate (Zijderveld et al., 2011, Lund et al., 2014, Patra \& Yu, 2014, Klop et al., 2016).

Phloroglucinol (1,3,5-trihydroxybenzene) is a simple phenolic compound that can be found naturally 
in the rumen as an intermediate product of polyphenol degradation, such as condensed tannin (CT) and hydrolysable tannin (HT) (McSweeney et al., 2001). Previous studies have shown that pure cultures of ruminal microorganisms are capable of degrading phloroglucinol to acetate with the use of $\mathrm{H}_{2}$ (Tsai et al., 1976, Patel et al., 1981 Krumholz \& Bryant, 1986). Supplementation of phloroglucinol can create competition for $\mathrm{H}_{2}$ between methanogen and phloroglucinol-degrading bacteria, which will decrease the amount of hydrogen available for methanogens to produce methane.

Previous in vivo study showed that phloroglucinol can redirect $[\mathrm{H}]$ toward acetate when methanogenesis is completely inhibited by addition of chloroform (Martinez-Fernandez et al., 2017). This showed that in the absence of methanogenesis which is a natural $[\mathrm{H}]$ sink and $\mathrm{H}_{2}$ pressure was high, phloroglucinol was able to redirect $[\mathrm{H}]$ toward acetate production. But, there was no effect found on methane production between chloroform only treatment and combination of chloroform and phloroglucinol treatment. Another study by Hierholtzer et al. (2012) found that phloroglucinol was able to decrease methane production from sludge collected from wastewater treatment. Sarwono et al. (2019) found that phloroglucinol could decrease methane production in different $\mathrm{F}: \mathrm{C}$ ratio, but there was little information regarding the role of phloroglucinol-degrading bacteria on $[\mathrm{H}]$ utilization. Therefore, the objective of this study was to investigate the effect of supplementation of phloroglucinol alone on methane production, fermentation profiles, and microbial population during in vitro rumen fermentation.

\section{MATERIALS AND METHODS}

\section{Animal and Sampling}

Animal handling was performed according to the Mie University guidelines for animal handling. Three male Friesland sheep were used as ruminal fluid donors. The animals were offered a daily ration consisting of $1 \mathrm{~kg}$ Italian rye grass hay and $360 \mathrm{~g}$ concentrate. The concentrate consisted of wheat bran, soy bean, and corn, in a 1:1:1 ratio on dry matter basis. The ration was divided into the same portion and offered twice daily at 10:00 and 17:00. Each animal was placed in an individual pen. Water and mineral block were available ad libitum. Rumen fluid was orally collected through a stomach tube just before the morning feeding and kept at $39^{\circ} \mathrm{C}$ during transportation to the laboratory.

\section{In Vitro Incubation}

Pooled ruminal fluid was strained through four layers of surgical gauze. The filtered fluid was mixed with McDougall buffer with ratio 1:2 (rumen fluid : buffer). The buffer was pre-warmed to $39^{\circ} \mathrm{C}$ and flushed with $\mathrm{N}_{2}$ gas. The diluted rumen fluid was used as inoculum. The composition of each treatment is described in Table 1. The substrate used consisted of $0.35 \mathrm{~g}$ of Italian rye grass hay, $0.30 \mathrm{~g}$ of wheat bran, and $0.35 \mathrm{~g}$ of corn. Each substrate was finely ground to pass through a $1 \mathrm{~mm}$ sieve using a Wiley mill. Phloroglucinol was dissolved in pure ethanol to $1 \mathrm{M}$. Five different doses of phloroglucinol $(2,4,6,8$, and $10 \mathrm{mmol} / \mathrm{L}$ media) were added to empty $120 \mathrm{~mL}$ serum bottles and dried overnight at $40^{\circ} \mathrm{C}$. After ethanol was evaporated, substrates were weighed into the bottle, and then $50 \mathrm{~mL}$ of media was dispensed under $\mathrm{N}_{2}$ gas. The serum bottle was tightly capped with a butyl rubber septum and aluminium cap. Then, it was incubated for $24 \mathrm{~h}$ at $39^{\circ} \mathrm{C}$ with shaking at $180 \mathrm{rpm}$ in a water bath. Each treatment consisted of three bottles, and three non-substrate bottles were also included in the incubation. The incubation was repeated three times on three separate days.

After $24 \mathrm{~h}$, all of the serum bottles were placed on ice to stop fermentation. The cumulative headspace gas was measured using a $100 \mathrm{~mL}$ glass syringe to determine total gas production. Methane production $(\mathrm{mL})$ was analysed using a gas chromatograph (GC-8A; Shimadzu Corporation, Kyoto, Japan) as described by Matsui et al. (2013). After gas analysis was completed, the culture fluid was transferred to a $50-\mathrm{mL}$ centrifugation tube and was centrifuged at $1000 \mathrm{~g}$ for $5 \mathrm{~min}$ at $4^{\circ} \mathrm{C}$ to separate the residue and the culture fluid. The culture fluid was transferred into a 2-mL tube and kept at $-30^{\circ} \mathrm{C}$ until analysis of SCFA, ammonia-N $\left(\mathrm{NH}_{3}-\mathrm{N}\right)$, and quantification of the microbial population. Meanwhile, the constant weight of the residue was measured to determine dry matter degradability. SCFAs in culture fluid were analysed by high-performance liquid chromatography (HPLC) as described by Uddin et al. (2010). $\mathrm{NH}_{3}-\mathrm{N}$ in the fluid was determined using the phenolhypochlorite method as described by Abrar et al. (2016).

A complete metabolic hydrogen $([\mathrm{H}])$ balance was estimated in accordance to Ungerfeld (2015). [2H] is total amount of reducing equivalent pairs produced. $[2 \mathrm{H}]_{\text {incorporated }}$ showed total amount of metabolic hydrogen incorporated into $\mathrm{H}_{2}, \mathrm{CH}_{4^{\prime}}$ propionate, and butyrate. $[2 \mathrm{H}]_{\text {recovery }}$ is percentage of hydrogen produced recovered in $\mathrm{CH}_{4}$, propionate, butyrate, and $\mathrm{H}_{2}$ divided by $[2 \mathrm{H}]$ produced. Molar amounts of methane for calculation $[\mathrm{H}]$ balance were calculated by using the following equation :

$$
p V=n R T
$$

where $p$ is the pressure of the gas assumed at $1 \mathrm{~atm}, V$ is the volume of the gas, $n$ is the number of moles of the gas, $R$ is ideal gas constant of 0.082 , and $T$ is the temperature assumed at $20^{\circ} \mathrm{C}$.

\section{DNA Extraction from the Rumen Fluid}

Microbial DNA was extracted from ruminal fluid using QIAamp DNA Stool Mini Kit according to the manufacture's instruction (Qiagen, Hilden, Germany). The extracted DNA was stored at $-30^{\circ} \mathrm{C}$ until analysis.

\section{Quantitation of the Microbial Population by Quantitative Real-Time PCR}

Cycle threshold $\left(\mathrm{C}_{\mathrm{T}}\right)$ of following 11 microbial populations, methanogen, F. succinogenes, Ruminococcus albus, Ruminococcus flavefaciens, Selonomonas ruminan- 
tium, Butyrivibrio fibrisolvens, genus Prevotella, genus Bacteroides, Streptococcus bovis, Eubacterium ruminantium, and genus Coprococcus were quantified by using realtime PCR to determine its relative abundance. Real-time PCR was conducted by using comparative $\Delta \Delta C_{T}$ method, as described by Schmittgen \& Livak (2008). Total bacteria was used as an endogenous control. Real-time PCR was conducted using a StepOnePlus ${ }^{\circledR}$ Real-Time PCR System (Applied Biosystems, Foster City, CA, USA).

Specific primers for target group and the amount used in this study are listed in Table 2. The reaction mixture $(20 \mu \mathrm{L})$ for real-time PCR consisted of $1 \mu \mathrm{L}$ of DNA template, $10 \mu \mathrm{L}$ of Thunderbird SYBR qPCR master mix (Toyobo Co. LTD., Osaka, Japan), $0.4 \mu \mathrm{L}$ 50x ROX, specific forward and reverse primer, and sterile Milli-Q water. The PCR condition of all target groups included one cycle of polymerase activation at $95^{\circ} \mathrm{C}$ for $1 \mathrm{~min}$, 40 cycles of denaturing at $95^{\circ} \mathrm{C}$ for $15 \mathrm{~s}$, and annealing and extension at $60^{\circ} \mathrm{C}$ for $1 \mathrm{~min}$ except for $R$. flavefaciens which include 40 cycles of denaturing at $95^{\circ} \mathrm{C}$ for $15 \mathrm{~s}$, annealing at $55^{\circ} \mathrm{C}$ for $15 \mathrm{~s}$, and extension at $60^{\circ} \mathrm{C}$ for 30 $\mathrm{s}$, and methanogen which include denaturing at $95^{\circ} \mathrm{C}$ for $15 \mathrm{~s}$, and annealing and extension at $60^{\circ} \mathrm{C}$ for $30 \mathrm{~s}$. Data of relative quantity of target groups were expressed by

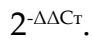

\section{Statistical Analysis}

Data were subjected to analysis of variance (ANOVA) according to a randomized complete block design. Different in vitro runs served as blocks in the ANOVA statistical model. Tukey's test was used to identify differences between treatment means when the ANOVA result showed a significant different. An effect was considered significant at the probability level of $P<0.05$, and $P<0.10$ was considered as a tendency to be significant. Polynomial contrasts were conducted to test the data for linear and quadratic trends. Statistical analysis was conducted using SPSS version 16.0 (SPSS Inc., Chicago, IL, USA).

Table 1. Composition of experimental in vitro batch cultures

\begin{tabular}{|c|c|c|c|c|c|c|}
\hline \multirow{2}{*}{ Material } & \multirow{2}{*}{ Control } & \multicolumn{5}{|c|}{ Phloroglucinol } \\
\hline & & $2 \mathrm{mmol} / \mathrm{L}$ & $4 \mathrm{mmol} / \mathrm{L}$ & $6 \mathrm{mmol} / \mathrm{L}$ & $8 \mathrm{mmol} / \mathrm{L}$ & $10 \mathrm{mmol} / \mathrm{L}$ \\
\hline Rye grass hay (g) & 0.35 & 0.35 & 0.35 & 0.35 & 0.35 & 0.35 \\
\hline Corn starch $(\mathrm{g})$ & 0.35 & 0.35 & 0.35 & 0.35 & 0.35 & 0.35 \\
\hline Wheat bran (g) & 0.30 & 0.30 & 0.30 & 0.30 & 0.30 & 0.30 \\
\hline Inoculum (mL) & 49.00 & 49.00 & 49.00 & 49.00 & 49.00 & 49.00 \\
\hline Phloroglucinol 1 M solution (mL) & - & 0.10 & 0.20 & 0.30 & 0.40 & 0.50 \\
\hline Distilled water $(\mathrm{mL})$ & 1.00 & 1.00 & 1.00 & 1.00 & 1.00 & 1.00 \\
\hline
\end{tabular}

Table 2. Primers used in recent study

\begin{tabular}{|c|c|c|c|c|}
\hline Target & Name & Sequence $\left(5^{\prime} \rightarrow 3^{\prime}\right)$ & $\begin{array}{l}\text { Amount added } \\
(\mu \mathrm{L})\end{array}$ & Reference \\
\hline \multirow[t]{2}{*}{ Total bacteria } & 1114-f & CGGCAACGAGCGCAACCC & 0.6 & Abrar et al. (2016) \\
\hline & $1275-\mathrm{r}$ & CCATTGTAGCACGTGTGTAGCC & 0.6 & \\
\hline \multirow[t]{2}{*}{ Ruminococcus albus } & Ra1281f & CССTAAAAGCAGTCTTAGTTCG & 0.5 & Lwin et al. (2012) \\
\hline & Ra1439r & ССTCCTTGCGGTTAGAACA & 0.5 & \\
\hline \multirow[t]{2}{*}{ Ruminococcus flavefaciens } & Rflf & GGACGATAATGACGGTACTT & 0.9 & Lwin et al. (2012) \\
\hline & Rflr & GCAATCYGAACTGGGACAAT & 0.9 & \\
\hline \multirow[t]{2}{*}{ Fibrobacter succinogenes } & Fsf & GGTATGGGATGAGCTTGC & 0.9 & Lwin et al. (2012) \\
\hline & Fsr & GCCTGCCCCTGAACTATC & 0.9 & \\
\hline \multirow[t]{2}{*}{ Methanogens } & q-mcrA-f & TTCGGTGGATCDCARAGRGC & 1.2 & Abrar et al. (2016) \\
\hline & q-mcrA-r & GBARGTCGWAWCCGTAGAATCC & 1.2 & \\
\hline \multirow[t]{2}{*}{ Selonomonas ruminantium } & SelRum 2F & CAATAAGCATTCCGCCTGGG & 0.45 & Mullins et al. (2013) \\
\hline & SelRum 2R & TTCACTCAATGTCAAGCCCTGG & 0.45 & \\
\hline \multirow[t]{2}{*}{ Butyrivibrio fibrisolvens } & ButFib 2F & ACCGCATAAGCGCACGGA & 0.2 & Mullins et al. (2013) \\
\hline & ButFib 2R & CGGGTCCATCTTGTACCGATAAAT & 0.2 & \\
\hline \multirow[t]{2}{*}{ Genus Prevotella } & PrevGen 4F & GGTTCTGAGAGGAAGGTCCCC & 0.2 & Mullins et al. (2013) \\
\hline & PrevGen $4 \mathrm{R}$ & TCCTGCACGCTACTTGGCTG & 0.2 & \\
\hline \multirow[t]{2}{*}{ Genus Bacteroides } & AllBac $296 f$ & GAGAGGAAGGTCCCCCAC & 0.2 & Ban-Tokuda et al. (2017) \\
\hline & AllBac $412 \mathrm{r}$ & CGCTACTTGGCTGGTTCAG & 3.6 & \\
\hline \multirow[t]{2}{*}{ Streptococcus bovis } & StrBov 2F & TTCCTAGAGATAGGAAGTTTCTTCGG & 8.8 & Mullins et al. (2013) \\
\hline & StrBov 2R & ATGATGGCAACTAACAATAGGGGT & 8.8 & \\
\hline \multirow[t]{2}{*}{ Eubacterium ruminantium } & EubRum 2F & CTCCCGAGACTGAGGAAGCTTG & 0.2 & Mullins et al. (2013) \\
\hline & EubRum 2R & GTCCATCTCACACCACCGGA & 0.2 & \\
\hline \multirow[t]{2}{*}{ Genus Coprococcus } & CopGenF & CATCCTGATGACGGTTTCTTAACC & 0.5 & Da Silva et al. (2018) \\
\hline & CopGenR & GTTGCGGGACTTAACCCA & 0.5 & \\
\hline
\end{tabular}




\section{RESULTS}

\section{In Vitro Rumen Fermentation}

Total gas production were significantly lowered 24 $\mathrm{h}$ after the addition of phloroglucinol $(\mathrm{p}<0.001$; Table $3)$. Compared with the control, gas production was decreased by $7.5 \%, 8.6 \%$, and $10.3 \%$ with the inclusion of 6,8 , and $10 \mathrm{mmol} / \mathrm{L}$ of phloroglucinol in the culture, respectively. There was a concentration-dependent and linear decrease $(p<0.001)$. Simultaneously, methane production $(\mathrm{mL})$ was significantly decreased $(\mathrm{p}<0.001)$ by $8.1 \%, 12.0 \%$, and $15.0 \%$ compared with the control, following supplementation with 6,8 , and $10 \mathrm{mmol} / \mathrm{L}$ phloroglucinol, respectively. However, supplementation of phloroglucinol had no significant effect on methane production when methane was presented as methane per digested dry matter $\left(\mathrm{CH}_{4} / \mathrm{DDM}\right)$.

Supplementing phloroglucinol significantly decreased dry matter degradability (DMD) $(p<0.001)$. Higher doses of phloroglucinol decreased DMD in a linear and concentration-dependent manner $(p<0.001)$. DMD was decreased by $7.3 \%, 8.9 \%$, and $13.4 \%$ compared with the control by the addition of 6,8 , and 10 $\mathrm{mmol} / \mathrm{L}$ of phloroglucinol, respectively.

Phloroglucinol did not affect ruminal pH after 24 $\mathrm{h}$ incubation; $\mathrm{pH}$ was between 5.72 and 5.77 after $24 \mathrm{~h}$. Phloroglucinol supplementation had neither a linear nor a quadratic effect on $\mathrm{pH}$. $\mathrm{NH}_{3}-\mathrm{N}$ was significantly abated by phloroglucinol supplementation $(\mathrm{p}<0.001)$. $\mathrm{NH}_{3}-\mathrm{N}$ decreased with increasing doses of phloroglucinol $(\mathrm{p}<0.001)$. Supplementation of 8 and $10 \mathrm{mmol} / \mathrm{L}$ of phloroglucinol decreased $\mathrm{NH}_{3}-\mathrm{N}$ by $62 \%$ and $70 \%$, respectively.

Total SCFA concentration was not significantly affected by phloroglucinol supplementation. Phloroglucinol had no linear or quadratic effect on total SCFA. Acetate proportion was increased significantly by phloroglucinol addition $(\mathrm{p}<0.001)$. The proportion of acetate to total SCFA increased concomitantly with increasing level of phloroglucinol $(\mathrm{p}<0.001)$, in a linear rather than a quadratic manner. The addition of 6,8 , or $10 \mathrm{mmol} / \mathrm{L}$ phloroglucinol increased the proportion of acetate by $8 \%, 12 \%$, and $16 \%$, respectively. The proportion of propionate was significantly decreased $(p<0.001)$. A linear decrease in propionate was observed $(p<0.001)$ with increasing doses of phloroglucinol. Additionally, the level of butyrate was not significantly affected by phloroglucinol supplementation.

Simultaneously, the acetate to propionate ratio increased significantly $(p<0.001)$ following phloroglucinol supplementation in a linear $(p<0.001)$ rather than a quadratic manner. The acetate to propionate ratio was significantly affected when phloroglucinol was supplemented at $6 \mathrm{mmol} / \mathrm{L}$ or higher.

\section{Micobial Population Density}

Phloroglucinol supplementation did not significantly affect the relative quantity of all target with the exception for Coprococcus ( $\mathrm{p}=0.006$; Table 4). Relative quantity of genus Coprococcus was linearly increased by increasing dose of phloroglucinol. A significant linear trend was also observed for Streptococcus bovis $(\mathrm{p}=0.024)$ and Eubacterium ruminantium ( $\mathrm{p}=0.018$ ).

\section{Hydrogen Balance}

Phloroglucinol supplementation did not have any significant effect on $[2 \mathrm{H}]_{\text {produced }}(P=0.061$; Table 5). Phloroglucinol significantly decreased $[2 \mathrm{H}]_{\text {incorporated }}$ and $[2 \mathrm{H}]_{\text {recovery. }}$ A significant linear and quadratic effect of phloroglucinol on $[2 \mathrm{H}]_{\text {incorporated }}$ was observed. A linear effect was also observed on $[2 \mathrm{H}]$

\section{DISCUSSION}

Phloroglucinol supplementation at $6 \mathrm{mmol} / \mathrm{L}$ or higher significantly lowered methane production in vitro in a dose-dependent manner. Our finding showed that

Table 3. In vitro methanogenesis and rumen fermentation supplemented by phloroglucinol

\begin{tabular}{|c|c|c|c|c|c|c|c|c|c|}
\hline \multirow{2}{*}{ Variables } & \multirow{2}{*}{ Control } & \multicolumn{5}{|c|}{ Phloroglucinol } & \multicolumn{3}{|c|}{ p-value } \\
\hline & & $2 \mathrm{mmol} / \mathrm{L}$ & $4 \mathrm{mmol} / \mathrm{L}$ & $6 \mathrm{mmol} / \mathrm{L}$ & $8 \mathrm{mmol} / \mathrm{L}$ & $10 \mathrm{mmol} / \mathrm{L}$ & Treatment & Linear & Quadratic \\
\hline $\begin{array}{l}\text { Total gas } \\
\text { production }(\mathrm{mL})\end{array}$ & $130.11 \pm 2.41^{\mathrm{a}}$ & $130.22 \pm 2.41^{a}$ & $\begin{array}{c}123.33 \pm \\
1.74^{\mathrm{ab}}\end{array}$ & $120.44 \pm 1.77^{b}$ & $118.89 \pm 1.40^{b}$ & $116.67 \pm 1.42^{b}$ & $<0.001$ & $<0.001$ & 0.583 \\
\hline $\begin{array}{l}\mathrm{CH}_{4} \text { production } \\
(\mathrm{mL})\end{array}$ & $16.72 \pm 0.32^{a}$ & $16.72 \pm 0.50^{\mathrm{ab}}$ & $15.90 \pm 0.29 \mathrm{abc}$ & $\begin{array}{l}15.36 \pm \\
0.24^{\text {bcd }}\end{array}$ & $14.71 \pm 0.25^{\mathrm{cd}}$ & $14.22 \pm 0.26^{\mathrm{d}}$ & $<0.001$ & $<0.001$ & 0.551 \\
\hline $\begin{array}{l}\mathrm{CH}_{4} \text { per digested } \\
\text { dry matter }(\mathrm{mL} / \mathrm{g})\end{array}$ & $34.21 \pm 0.48$ & $34.60 \pm 0.95$ & $33.935 \pm 0.47$ & $34.21 \pm 0.31$ & $33.02 \pm 0.46$ & $33.94 \pm 0.61$ & 0.538 & 0.242 & 0.926 \\
\hline $\begin{array}{l}\text { Dry matter } \\
\text { degradability (\%) }\end{array}$ & $48.86 \pm 0.37^{a}$ & $48.31 \pm 0.37^{\mathrm{ab}}$ & $46.86 \pm 0.50^{\mathrm{bc}}$ & $44.90 \pm 0.57^{c d}$ & $44.54 \pm 0.39^{d}$ & $41.94 \pm 0.57 \mathrm{e}$ & $<0.001$ & $<0.001$ & 0.178 \\
\hline $\mathrm{pH}$ & $5.77 \pm 0.23$ & $5.75 \pm 0.25$ & $5.77 \pm 0.15$ & $5.76 \pm 0.15$ & $5.75 \pm 0.16$ & $5.72 \pm 0.18$ & 0.434 & 0.088 & 0.307 \\
\hline $\mathrm{NH}_{3}-\mathrm{N}(\mathrm{mg} / \mathrm{dL})$ & $4.64 \pm 0.27^{a}$ & $4.80 \pm 0.58^{\mathrm{a}}$ & $3.62 \pm 0.36^{\mathrm{ab}}$ & $2.48 \pm 0.33^{\mathrm{bc}}$ & $1.76 \pm 0.38^{c}$ & $1.39 \pm 0.44^{c}$ & $<0.001$ & $<0.001$ & 0.102 \\
\hline $\begin{array}{l}\text { Total SCFA } \\
(\mathrm{mmol} / \mathrm{L})\end{array}$ & $124.98 \pm 1.78$ & $129.84 \pm 1.89$ & $126.40 \pm 1.25$ & $125.39 \pm 1.21$ & $125.14 \pm 1.15$ & $126.67 \pm 1.07$ & 0.172 & 0.581 & 0.765 \\
\hline \multicolumn{10}{|c|}{ Percentage to total SCFA } \\
\hline Acetate $(\%)$ & $60.91 \pm 0.23^{a}$ & $61.93 \pm 0.18^{\mathrm{ab}}$ & $62.61 \pm 0.25^{\mathrm{bc}}$ & $62.71 \pm 0.33^{\mathrm{bc}}$ & $63.36 \pm 0.31^{\mathrm{cd}}$ & $64.41 \pm 0.46^{\mathrm{d}}$ & $<0.001$ & $<0.001$ & 0.989 \\
\hline Propionate (\%) & $27.47 \pm 0.24^{\mathrm{a}}$ & $26.48 \pm 0.21^{\mathrm{ab}}$ & $25.97 \pm 0.28^{b}$ & $25.65 \pm 0.34^{b}$ & $25.02 \pm 0.41^{\mathrm{bc}}$ & $24.11 \pm 0.55^{c}$ & $<0.001$ & $<0.001$ & 0.987 \\
\hline Butyrate (\%) & $11.63 \pm 0.08$ & $11.59 \pm 0.19$ & $11.43 \pm 0.18$ & $11.65 \pm 0.10$ & $11.62 \pm 0.13$ & $11.48 \pm 0.15$ & 0.859 & 0.724 & 0.992 \\
\hline Acetate : Propionate & $2.22 \pm 0.03^{\mathrm{a}}$ & $2.34 \pm 0.02^{\mathrm{ab}}$ & $2.41 \pm 0.03^{\mathrm{abc}}$ & $2.45 \pm 0.04^{\mathrm{bc}}$ & $2.54 \pm 0.05^{\mathrm{cd}}$ & $2.68 \pm 0.08^{\mathrm{d}}$ & $<0.001$ & $<0.001$ & 0.665 \\
\hline
\end{tabular}

Note: Values presented are mean \pm standard error of mean $(n=9)$. Means in the same row with different superscripts differ significantly $(\mathrm{p} \leq 0.05)$. 
Table 4. Relative microbial population density as expressed by $2^{-\Delta \Delta \mathrm{CT}_{\mathrm{T}}}$ supplemented by phloroglucinol

\begin{tabular}{|c|c|c|c|c|c|c|c|c|c|}
\hline \multirow{2}{*}{ Species } & \multirow{2}{*}{ Control } & \multicolumn{5}{|c|}{ Phloroglucinol } & \multicolumn{3}{|c|}{ p-value } \\
\hline & & $2 \mathrm{mmol} / \mathrm{L}$ & $4 \mathrm{mmol} / \mathrm{L}$ & $6 \mathrm{mmol} / \mathrm{L}$ & $8 \mathrm{mmol} / \mathrm{L}$ & $10 \mathrm{mmol} / \mathrm{L}$ & Treatment & Linear & Quadratic \\
\hline Ruminococcus albus & $1.00 \pm 0.00$ & $1.52 \pm 0.51$ & $0.87 \pm 0.03$ & $0.82 \pm 0.13$ & $0.74 \pm 0.12$ & $0.92 \pm 0.21$ & 0.299 & 0.242 & 0.694 \\
\hline $\begin{array}{l}\text { Ruminococcus } \\
\text { flaveciens }\end{array}$ & $1.00 \pm 0.00$ & $0.84 \pm 0.14$ & $0.82 \pm 0.18$ & $0.96 \pm 0.14$ & $1.02 \pm 0.09$ & $0.88 \pm 0.19$ & 0.857 & 0.936 & 0.591 \\
\hline $\begin{array}{l}\text { Fibrobacter } \\
\text { succinogenes }\end{array}$ & $1.00 \pm 0.00$ & $1.17 \pm 0.26$ & $1.24 \pm 0.15$ & $1.10 \pm 0.09$ & $0.89 \pm 0.17$ & $0.72 \pm 0.06$ & 0.214 & 0.126 & 0.038 \\
\hline Methanogens & $1.00 \pm 0.00$ & $1.29 \pm 0.14$ & $1.38 \pm 0.11$ & $1.31 \pm 0.19$ & $1.41 \pm 0.26$ & $1.36 \pm 0.14$ & 0.539 & 0.113 & 0.333 \\
\hline $\begin{array}{l}\text { Selonomonas } \\
\text { ruminantium }\end{array}$ & $1.00 \pm 0.00$ & $1.06 \pm 0.17$ & $1.01 \pm 0.12$ & $1.28 \pm 0.21$ & $1.42 \pm 0.43$ & $1.04 \pm 0.28$ & 0.253 & 0.119 & 0.513 \\
\hline $\begin{array}{l}\text { Butyrivibrio } \\
\text { fibrisolvens }\end{array}$ & $1.00 \pm 0.00$ & $1.06 \pm 0.05$ & $0.98 \pm 0.02$ & $0.87 \pm 0.04$ & $0.87 \pm 0.11$ & $0.95 \pm 0.11$ & 0.406 & 0.175 & 0.915 \\
\hline Genus Prevotella & $1.00 \pm 0.00$ & $1.07 \pm 0.04$ & $1.03 \pm 0.06$ & $0.97 \pm 0.04$ & $1.09 \pm 0.06$ & $0.96 \pm 0.09$ & 0.493 & 0.738 & 0.385 \\
\hline Genus Bacteroides & $1.00 \pm 0.00$ & $1.06 \pm 0.02$ & $0.96 \pm 0.05$ & $0.96 \pm 0.03$ & $1.06 \pm 0.03$ & $0.92 \pm 0.07$ & 0.125 & 0.256 & 0.378 \\
\hline Streptococcus bovis & $1.00 \pm 0.00$ & $0.98 \pm 0.32$ & $0.81 \pm 0.12$ & $1.09 \pm 0.10$ & $1.96 \pm 0.58$ & $1.69 \pm 0.15$ & 0.850 & 0.024 & 0.157 \\
\hline $\begin{array}{l}\text { Eubacterium } \\
\text { ruminantium }\end{array}$ & $1.00 \pm 0.00$ & $1.25 \pm 0.07$ & $1.15 \pm 0.04$ & $1.33 \pm 0.21$ & $1.47 \pm 0.21$ & $1.50 \pm 0.21$ & 0.218 & 0.018 & 0.896 \\
\hline Genus Coprococcus & $1.00 \pm 0.00^{\mathrm{a}}$ & $1.75 \pm 0.44^{\mathrm{ab}}$ & $1.68 \pm 0.09^{\mathrm{ab}}$ & $2.29 \pm 0.32^{\mathrm{b}}$ & $2.89 \pm 0.25^{\mathrm{b}}$ & $2.31 \pm 0.27^{\mathrm{b}}$ & 0.006 & $<0.001$ & 0.374 \\
\hline
\end{tabular}

Note: Values presented are mean \pm standard error of mean $(n=9)$. Means in the same row with different superscripts differ significantly ( $\leq \leq .05)$.

Table 5. Metabolic hydrogen balance

\begin{tabular}{|c|c|c|c|c|c|c|c|c|c|}
\hline \multirow{2}{*}{ Variables } & \multirow{2}{*}{ Control } & \multicolumn{5}{|c|}{ Phloroglucinol } & \multicolumn{3}{|c|}{ p-value } \\
\hline & & $2 \mathrm{mmol} / \mathrm{L}$ & $4 \mathrm{mmol} / \mathrm{L}$ & $6 \mathrm{mmol} / \mathrm{L}$ & $8 \mathrm{mmol} / \mathrm{L}$ & $10 \mathrm{mmol} / \mathrm{L}$ & Treatment & Linear & Quadratic \\
\hline$[2 \mathrm{H}]_{\text {produced }}(\mathrm{mmol})$ & $12.232 \pm 0.158$ & $12.768 \pm 0.183$ & $12.441 \pm 0.102$ & $12.390 \pm 0.098$ & $12.400 \pm 0.088$ & $12.591 \pm 0.089$ & 0.070 & 0.361 & 0.334 \\
\hline$[2 \mathrm{H}]_{\text {incorporated }}(\mathrm{mmol})$ & $7.671 \pm 0.060^{\mathrm{ab}}$ & $7.729 \pm 0.143^{a}$ & $7.380 \pm 0.058^{\mathrm{bc}}$ & $7.236 \pm 0.054^{\mathrm{cd}}$ & $7.036 \pm 0.118^{\mathrm{de}}$ & $6.878 \pm 0.061^{\mathrm{e}}$ & $<0.001$ & $<0.001$ & 0.023 \\
\hline$[2 \mathrm{H}]_{\text {recovery }}(\%)$ & $62.758 \pm 0.600^{\mathrm{a}}$ & $60.519 \pm 0.539^{b}$ & $59.295 \pm 0.338^{\mathrm{bc}}$ & $58.410 \pm 0.262^{\mathrm{cd}}$ & $56.755 \pm 0.331^{\mathrm{d}}$ & $54.634 \pm 0.430^{\mathrm{e}}$ & $<0.001$ & $<0.001$ & 0.084 \\
\hline
\end{tabular}

Note: Values presented are mean \pm standard error of mean $(n=9)$. Means in the same row with different superscripts differ significantly ( $\leq \leq 0.05)$.

phloroglucinol can inhibit methane production in the presence of methanogenesis. Our finding is in agreement to previous study that found methane decrease by phloroglucinol supplementation (Sarwono et al., 2019). Our results confirmed previous findings suggesting that simple phenolics such as cinnamic, caffeic, ferulic acid, $p$-coumaric, and benzoic acid can decrease methane production (Jayanegara, 2010).

Phenolic compounds might decrease methane production from the rumen through inhibition of methanogen growth and/or through inhibition of carbohydrate digestion, thus decreasing $\mathrm{H}_{2}$ production (Jayanegara et al., 2015). In the present study, phloroglucinol had no effect on the methanogen population density. This showed that the decrease of methane production by phloroglucinol was not directly related to the inhibition of methanogen growth. The decrease of methane production by phloroglucinol was presumably related to the inhibition of nutrient digestibility by ruminal microbes, particularly carbohydrate digestion, thus decreasing the amount of $\mathrm{H}_{2}$ available for methane production. The latter mechanism was reflected in our study.

Phloroglucinol supplementation significantly suppressed total gas production and DMD, indicating that it might suppress carbohydrate fermentation. Phloroglucinol supplementation also suppressed $\mathrm{NH}_{3}-\mathrm{N}$ production after $24 \mathrm{~h}$. High levels of phloroglucinol (10 mmol/L) decreased $\mathrm{NH}_{3}-\mathrm{N}$ by $72 \%$. This finding showed that phloroglucinol might inhibit nutrient degradation. This finding is in agreement with Kisworo et al. (2017) that found lowered gas production, methane, and $\mathrm{NH}_{3}-\mathrm{N}$ by phenolic compound from solid herbal waste. The possible explanation is that phloroglucinol contain three free hydroxyl groups in its chemical structure which allow interaction with nutrients, such as carbohydrate, fibre, and protein through strong hydrogen bond formation resulting in complexes (Dobreva et al., 2011). Our finding is in agreement with previous study which suggest that phenolic compound might inhibit fibre and protein digestion (McSweeney et al., 2001, Jayanegara \& Palupi, 2010). Of particular interest, inhibition of fibre digestion could lead to lower $\mathrm{H}_{2}$ and $\mathrm{CO}_{2}$ gas production, which are the main precursors for methane production. Therefore, any decrease in carbohydrate and protein digestibility by phloroglucinol could indirectly decrease methane production.

Interestingly, although phloroglucinol was likely to decrease carbohydrate digestion, phloroglucinol supplementation did not affect total SCFA production, which is the main fermentation product of carbohydrate digestion in the rumen. This might be related to changes in the level of SCFA proportion following phloroglucinol supplementation. In this study, The SCFA concentration shifted to acetate at the expense of propionate; however, butyrate was not affected by phloroglucinol. This showed that phloroglucinol might redirect rumen fermentation from methane production to acetate production. Our finding showed that treatment of 10 $\mathrm{mmol} / \mathrm{L}$ phloroglucinol produced $0.274 \mathrm{mmol}$ of acetate more than control, which is lower than predicted moles of acetate $(1 \mathrm{mmol})$ produced from phloroglucinol 
degradation in rumen ( 1 molecule of phloroglucinol= 2 molecules of acetate +2 molecules of $\mathrm{CO}_{2}$ (Tsai et al., 1976). This finding showed that phloroglucinol could redirect $[\mathrm{H}]$ toward acetate production under functioning methanogenesis in vitro. Meanwhile, lower amount of acetate production than prediction might showed that phloroglucinol was not only used as a hydrogen sink but also was used to inhibit nutrient digestion.

The change of rumen fermentation by phloroglucinol to acetate production was accompanied by simultaneous increment of genus Coprococcus relative quantity. This finding showed that Coprococcus plays an important role on phloroglucinol metabolism in the rumen. Increasing genus Coprococcus relative quantity might partly related to reduction of methane production as it increases the competition for $[\mathrm{H}]$ utilization with methanogen. This finding was in agreement with the study of Martinez-Fernandez et al. (2017). An increase of acetate production was observed simultaneously with an increase of several operational taxonomic units (OTUs) assigned to Coprococcus spp. when phloroglucinol was added under methanogenesis inhibited. Tsai \& Jones (1975) found that genus Coprococcus were able to metabolize phloroglucinol.

A complete metabolic hydrogen balance showed that phloroglucinol did not have any significant effect on $[2 \mathrm{H}]_{\text {produced }}$ but significantly lowered $[2 \mathrm{H}]_{\text {incorporated }}$ and $[2 \mathrm{H}]_{\text {recovery }}$ although there was a possible redirection of $[\mathrm{H}]$ toward acetate by phloroglucinol addition. This finding was similar to the previous study by Ungerfeld (2015) that found a significant decrease of $[2 \mathrm{H}]_{\text {in }}$ and $[2 \mathrm{H}]_{\text {recovery }}$ when methanogenesis is inhibited. This might be related to the formula used to calculate the hydrogen balance which did not include $[\mathrm{H}]$ sink other than main fermentation products such as propionate, butyrate, and $\mathrm{H}_{2}$. This include redirection of $[\mathrm{H}]$ to acetate by phloroglucinol supplementation.

\section{CONCLUSION}

Phloroglucinol was able to significantly lower methane production by inhibiting nutrient digestibility and by possible redirection of rumen fermentation to acetate production in the rumen. Genus Coprococcus could be an important actor for phloroglucinol metabolism in the rumen

\section{CONFLICT OF INTEREST}

The authors certify that there is no conflict of interest with any financial organization regarding the material discussed in the manuscript.

\section{ACKNOWLEDGEMENT}

Real-time PCR was carried out at The Life Science Research Center (Center for Molecular Biology and Genetics), Mie University (Tsu, Japan). Special gratitude to Hani Sukmawati for her assistance in collecting ruminal fluid.

\section{REFERENCES}

Abrar, A., M. Kondo, T. Kitamura, T. Ban-Tokuda, \& H. Matsui. 2016. Effect of supplementation of rice bran and fumarate alone or in combination on in vitro rumen fermentation, methanogenesis and methanogens. Anim. Sci. J. 87:398-404. https://doi.org/10.1111/asj.12431

Ban-Tokuda, T., S. Maekawa, T. Miwa, S. Ohkawara, \& H. Matsui. 2017. Changes in faecal bacteria during fattening in finishing swine. Anaerobes 47:188-193. https://doi. org/10.1016/j.anaerobe.2017.06.006

Broucek, J. 2018. Options to methane production abatement in ruminants: A review. J. Anim. Plant Sci. 28:348-364.

Da Silva, H. E., A. Teterina, E. M. Cornelli, A. Taibi, B. M. Arendt, S. E. Fischer, W. Lou, \& J. P. Allard. 2018. Nonalcoholic fatty liver disease is associated with dysibiosis independent of body mass indes and insulin resistance. Sci. Rep. 8(1466): 1-12. https://doi.org/10.1038/ s41598-018-19753-9

Dobreva, M. A., R. A. Frazier, I. Mueller-Harvey, L. A. Clifton, A. Gea, \& R. J. Green. 2011. Binding of pentagalloyl glucose to two globular proteins occurs via multiple surface sites. Biomacromolecules 12:710-715. https://doi. org/10.1021/bm101341s

Hierholtzer, A., L. Chatellard, M. Kierans, J. C. Akunna, \& P. J. Collier. 2012. The impact and mode of action of phenolic compounds extracted from brown seaweed on mixed anaerobic microbial cultures. J. Appl. Microbiol., 114:964-973. https://doi.org/10.1111/jam.12114

Jayanegara, A. 2010. Ruminal methane production on simple phenolic acids addition in in vitro gas production method. Med. Pet. 32:53-62.

Jayanegara, A., \& E. Palupi. 2010. Condensed tannin effects on nitrogen digestion in ruminants: a meta-analysis from in vitro and in vivo studies. Med. Pet. 33:176-181. https://doi. org/10.5398/medpet.2010.33.3.176

Jayanegara, A., G. Goel, H. P. S. Makkar, \& K. Becker. 2015. Divergence between purified hydrolysable and condensed tannin effects on methane emission, rumen fermentation and microbial population in vitro. Anim. Feed Sci. Tech. 209:60-68. https://doi.org/10.1016/j.anifeedsci.2015.08.002

Johnson, K. A., \& D. E. Johnson. 1995. Methane emissions from cattle. J. Anim. Sci, 73:2483-2492. https://doi. org/10.2527/1995.7382483x

Kisworo, A. N., A. Agus, Kustantinah, \& B. Suwingyo. 2017. Physicochemical characteristics, in vitro fermentation indicators, gas production kinetics, and degradability of solid herbal waste as alternative feed source for ruminants. Med. Pet. 40:101-110. https://doi.org/10.5398/ medpet.2017.40.2.101

Klop, G., B. Hatew, A. Bannink, \& J. Dijkstra. 2016. Feeding nitrate and docosahexaenoic acid affects enteric methane production and milk fatty acid composition in lactating dairy cows. J. Dairy Sci. 99:1161-1172. https://doi. org/10.3168/jds.2015-10214

Knapp, J. R., G. L. Laur, P. A. Vadas, W. P. Weiss, \& J. M. Tricarico. 2014. Enteric methane in dairy cattle production: Quantifying the opportunities and impact of reducing emissions. J. Dairy Sci. 97:3231-3261. https://doi. org $/ 10.3168 /$ jds.2013-7234

Krumholz, L. R., \& M. P. Bryant. 1986. Eubacterium oxidoreducens sp. nov. requiring $\mathrm{H} 2$ or formate to degrade gallate, pyrogallol, phloroglucinol and quercetin. Arch. Microbiol. 144:8-14. https://doi.org/10.1007/BF00454948

Lan, W. \& C. Yang. 2019. Ruminal methane production: Associated microorganisms and the potential of applying hydrogen-utilizing bacteria for mitigation. Sci. Total Environ. 654:1270-1283. https://doi.org/10.1016/j. scitotenv.2018.11.180 
Li, Z., N. Liu, Y. Cao, C. Jin, F. Li, C. Cai, \& J. Yao. 2018. Effects of fumaric acid supplementation on methane production and rumen fermentation in goats fed diets varying in forage and concentrate particle size. J. Anim. Sci. Biotechnol. 9:21. https://doi.org/10.1186/s40104-018-0235-3

Lund, P., R. Dahl, H. J. Yang, A. L. F. Hellwing, B. B. Cao, \& M. R. Weisbjerg. 2014. The acute effect of addition of nitrate on in vitro and in vivo methane emission in dairy cows. Anim. Prod. Sci. 54:1432-1435. https://doi.org/10.1071/ AN14339

Lwin, K. O., M. Kondo, T. Ban-Tokuda, R. M. Lapitan, A. N. Del-Barrio, T. Fujihara, \& H. Matsui. 2012. Ruminal fermentation and microbial ecology of buffaloes and cattle fed the same diet. Anim. Sci. J. 83:767-776. https://doi. org/10.1111/j.1740-0929.2012.01031.x

Martin, C., D. Morgavi, \& M. Doreau. 2010. Methane mitigation in ruminants: From microbe to the farm scale.Animal 4:351-365. https://doi.org/10.1017/S1751731109990620

Martinez-Fernandez, G., S. E. Denman, J. Cheung, \& C. S. McSweeney. 2017. Phloroglucinol degradation in the rumen promotes the capture of excess hydrogen generated from methanogenesis inhibition. Front. Microbiol. 8:1871. https://doi.org/10.3389/fmicb.2017.01871

Matsui, H., H. Wakabayashi, N. Fukushima, K. Ito, A. Nishikawa, R. Yoshimi, Y. Ogawa, S. Yoneda, T. BanTokuda, \& M. Wakita. 2013. Effect of raw rice bran supplementation on rumen methanogen population density and in vitro rumen fermentation. Grassl. Sci. 59:129-134. https://doi.org/10.1111/grs.12023

McSweeney, C. S., B. Palmer, D. M. McNeill, \& D. O. Krause. 2001. Microbial interactions with tannins: nutritional consequences for ruminants. Anim. Feed Sci. Technol. 91:8393. https://doi.org/10.1016/S0377-8401(01)00232-2

Mullins, C. R., L. K. Mamedova, A. J. Carpenter, Y. Ying, M. S. Allen, I. Yoon, \& B. J. Bradford. 2013. Analysis of rumen microbial populations in lactating dairy cattle fed diets varying in carbohydrate profiles and Saccharomyces cerevisiae fermentation product. J. Dairy Sci. 96:5872-5881. https://doi.org/10.3168/jds.2013-6775

Myhre, G., D. Shindell, F. -M. Bréon, W. Collins, J. Fuglestvedt, J. Huang, D. Koch, J. -F. Lamarque, D. Lee, B. Mendoza, T. Nakajima, A. Robock, G. Stephens, T. Takemura, \& H. Zhang. 2013. Anthropogenic and Natural Radiative Forcing. In: Stocker, T. F., D. Qin, G. -K. Plattner, M. Tignor, S. K. Allen, J. Doschung, A. Nauels, Y. Xia, V. Bex, \& P. M. Midgley (Eds.). Climate Change 2013: The Physical Science Basis. Contribution of Working Group I to the Fifth Assessment Report of the Intergovernmental Panel on Climate Change. Cambridge University Press, Cambridge. pp. 659-740.

Patel, T. R., K. G. Jure, \& G. A. Jones. 1981. Catabolism of phloroglucinol by the rumen anaerobe Coprococcus. Appl. Environ. Microb. 42:1010-1017.
Patra, A. K., \& Z. Yu. 2012. Effects of essential oils on methane production and fermentation by, and abundance and diversity of, rumen microbial populations. Appl. Environ. Microbiol. 78:4271-4280. https://doi.org/10.1128/ AEM.00309-12

Sarwono, K. A., M. Kondo, T. Ban-Tokuda, A. Jayanegara, \& H. Matsui. 2019. Effects of phloroglucinol and the forage:concentrate ratio on methanogenesis, in vitro rumen fermentation, and microbial population density. Adv. Anim. Vet. Sci. 7:164-171. https://doi.org/10.17582/journal. aavs/2019/7.3.164.171

Schmittgen, T. D., \& K. J. Livak. 2008. Analyzing real-time PCR data by the comparative CT method. Nat. Protoc. 3:1101-1108.

Smith, P., H. Clark, H. Dong, E. A. Elsiddig, H. Haberl, R. Harper, J. House, M. Jafari, O. Masera, C. Mbow, N. H. Ravindranath, C. W. Rice, C. Roble Do Abad, A. Romanovskaya, F. Sperling, \& F. Tubiello. 2014. Chapter 11 - Agriculture, forestry and other land use (AFOLU). Climate Change 2014: Mitigation of Climate Change. IPCC Working Group III Contribution to AR5. Cambridge University Press, Cambridge.

Song, M. K., X. Z. Li, Y. K. Oh, C. K. Lee, \& Y. Hyun. 2011. Control of methane emission in ruminants and industrial application of biogas from livestock manure in korea. Asian-Aust. J. Anim. Sci. 24:130-136. https://doi. org/10.5713/ajas.2011.r.02

Tsai, C. G. \& G. A. Jones. 1975. Isolation and identification of rumen bacteria capable of anaerobic phloroglucinol degradation. Can. J. Microbiol. 21:794-801. https://doi. org/10.1139/m75-117

Tsai, C. G., D. M. Gates, W. M. Ingledew, \& G. A. Jones. 1976. Products of anaerobic phloroglucinol degradation by Coprococcus sp. Pe15. Can. J. Microbiol. 22:159-64. https:// doi.org/10.1139/m76-022

Uddin, M. K., M. Kondo, J. Kita, H. Matsui, S. Karita, M. Goto. 2010. Effect of supplementation of soy sauce cake and vinegar brewer's cake with total mixed ration silage-based diet on nutrient utilization by Holstein steers. J. Food Agric. Environ. 8:282-287.

Ungerfeld, E. M. 2015. Shifts in metabolic hydrogen sinks in the methanogenesis-inhibited ruminal fermentation: a metaanalysis. Front. Microbiol. 6:37. https://doi.org/10.3389/ fmicb.2015.00538

Zijderveld, van S.M., B. Fonken, J. Dijkstra, W.J. Gerrits, H.B. Perdok, W. Fokkink, \& J.R. Newbold. 2011. Effects of a combination of feed additives on methane production, diet digestibility, and animal performance in lactating dairy cows. J. Dairy Sci. 94:1445-1454. https://doi.org/10.3168/ jds.2010-3635 